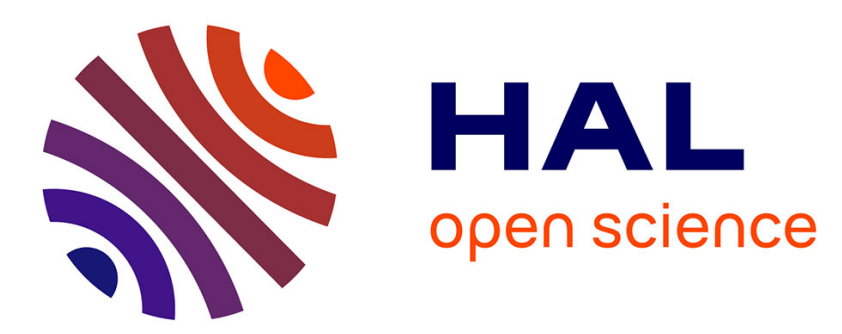

\title{
Revisiting the discrete planar Laplacian: exact results for the lattice Green function and continuum limit
}

Malik Mamode

\section{To cite this version:}

Malik Mamode. Revisiting the discrete planar Laplacian: exact results for the lattice Green function and continuum limit. The European Physical Journal Plus, 2021, 136 (4), 10.1140/epjp/s13360-02101439-3 . hal-03204762

\section{HAL Id: hal-03204762 \\ https://hal.science/hal-03204762}

Submitted on 21 Apr 2021

HAL is a multi-disciplinary open access archive for the deposit and dissemination of scientific research documents, whether they are published or not. The documents may come from teaching and research institutions in France or abroad, or from public or private research centers.
L'archive ouverte pluridisciplinaire HAL, est destinée au dépôt et à la diffusion de documents scientifiques de niveau recherche, publiés ou non, émanant des établissements d'enseignement et de recherche français ou étrangers, des laboratoires publics ou privés. 


\title{
Revisiting the discrete planar Laplacian: exact results for the lattice Green function and continuum limit
}

\author{
MALIK MAMODE* \\ Department of Physics, Laboratoire PIMENT, University of La Réunion (France)
}

\begin{abstract}
The paper deals with the discrete Laplacian on a uniform infinite square lattice. The definition of its fundamental solution or lattice Green function (LGF) is clarified as the Fourier coefficients of a certain generalized periodic function $g$. Such a functional must be regularized and gives the LGF up to a constant equal to $\langle g\rangle$, the mean value of $g$. For $\langle g\rangle=0$, the LGF may be expressed in an exact analytic form in terms of hypergeometric and gamma functions. The continuum limit of the LGF is finally studied requiring an appropriate renormalization of $\langle g\rangle$ in order to obtain the logarithmic Coulomb potential.
\end{abstract}

\section{Introduction}

Lattice Green functions arise in a wide variety of theoretical problems covering e.g. the lattice gauge theories [1, 2], lattice models of statistical physics [3] as well as the study of random walks $[4,5]$ or the calculation of two-point resistances of resistor networks $[6,7]$. They are also central in numerical analysis of partial differential equations for which the discretization of the continuum on periodic lattices is for instance frequently used to deal with point singularities before considering a renormalization procedure when the continuum limit is in fine taken $[8,9,10]$. In this respect, the Poisson equation and the use of the related lattice Green function for the Laplacian operator are emblematic of lattice discretization technique and constitute a toy model for implementing the lattice regularization of Coulombian potentials singularities.

The present work proposes to revisit the planar Poisson function and its discretization on an uniform infinite square lattice $\Lambda=\mathbb{Z}^{2}$. The justification for such a restriction should be clearer below. As is known, the lattice Green function (LGF) $G[n, m]$ on $\Lambda$ is thus defined as the isotropic solution of the partial difference equation

$$
\Delta G[n, m]:=G[n+1, m]+G[n-1, m]+G[n, m+1]+G[n, m-1]-4 G[n, m]=-\delta[n, m] \quad, \quad(n, m) \in \Lambda
$$

where $\delta$ is the Kronecker delta

$$
\delta[n, m]=\left\{\begin{array}{ll}
1 & n=m=0 \\
0 & \text { otherwise }
\end{array} .\right.
$$

The operator $\Delta$ is a finite-difference representation of the planar Laplacian.

Such a solution is unique (up to a constant) and the term isotropic means it must demonstrate the properties of symmetry and uniformity of the square lattice. The LGF is usually found using a discrete Fourier transform and may be interpreted as Fourier coefficients of a generalized periodic function on the flat 2-torus. A detailed review of these results is given in Section 1 (and the generalization for any other dimensionality $d$ should be straightforward).

Except in one dimension where the LGF can be derived exactly as

$$
G[n]=-\frac{|n|}{2}+k, n \in \mathbb{Z}, k \text { arbitrary constant, }
$$

the analytic calculation of the LGF $G[n, m]$ from its Fourier integral representation is very difficult and de facto no general closed-form result exists to date apart a few partial results $[7,11,12]$. Unfortunately, this is also the case for higher dimensions (see e.g. [13]). In this work, we show in Section 2 that the exact expression of the planar LGF $G[n, m]$ for any $(n, m) \in \Lambda$ can be actually derived in terms of hypergeometric and gamma functions.

*malik.mamode@univ-reunion.fr 
The continuum limit of the LGF is finally examined in Section 3. In one dimension, (2) clearly shows that it is the exact discretized counterpart for the fundamental solution of the Laplacian on the real line whatever the unit length chosen i.e. the lattice spacing. Indeed, recall that the solution of $\Delta \mathcal{G}=-\delta(x)$, $\delta$ is the Dirac distribution at origin, is the even function $\mathcal{G}(x)=-|x| / 2+k, k$ an arbitrary constant. The situation is quite different in the 2-dimensional case because of the logarithmic singularity and the scale invariance of the radially symmetric potential $\mathcal{G}(\mathbf{r})=-(1 / 2 \pi) \log (|\mathbf{r}| / L), L$ an arbitrary length, solution of $\Delta \mathcal{G}=-\delta(\mathbf{r}), \mathbf{r}=(x, y) \in \mathbb{R}^{2}$. Since the LGF $G[n, m]$ is finite everywhere on the square lattice, a specific renormalization procedure is needed to recover the Coulomb logarithmic potential in the continuum limit. All this sheds particular light on specific features of the infinite square lattice and the continuous plane which do not exist in no other dimensionality.

\section{Lattice Green function : definition}

Assuming that $G[n, m]$ increases no faster than some power $n^{k} m^{l}$ as $|n|+|m| \rightarrow+\infty$, the double trigonometric series

$$
\sum_{(n, m) \in \mathbb{Z}^{2}} G[n, m] e^{i\left(n \theta_{1}+m \theta_{2}\right)}
$$

converges in the sense of generalized functions to $g\left(\theta_{1}, \theta_{2}\right)[14]$ such that, according to (1),

$$
g\left(\theta_{1}, \theta_{2}\right)\left(2-\cos \theta_{1}-\cos \theta_{2}\right)=\frac{1}{2}
$$

It is worth to notice right away that the evenness of $g$ in both variables and its symmetry property $g\left(\theta_{1}, \theta_{2}\right)=$ $g\left(\theta_{2}, \theta_{1}\right)$ agree with the reflection and symmetry properties of the LGF i.e.

$$
G[n, m]=G[ \pm n, \pm m] \quad, \quad G[n, m]=G[m, n] .
$$

By division, one obtains up to a constant multiple of a 2-dimensional Dirac comb $\delta\left(\theta_{1}, \theta_{2}\right)$, the generalized function on the flat 2-torus $\mathbb{R}^{2} /(2 \pi \mathbb{Z})^{2}$,

$$
g\left(\theta_{1}, \theta_{2}\right)=\frac{1}{2} \frac{1}{2-\cos \theta_{1}-\cos \theta_{2}}
$$

defined for any test-function $\varphi\left(\theta_{1}, \theta_{2}\right)$ on the torus by the following regularized double integral owing to the singularity at the origin,

$$
\left\langle g\left(\theta_{1}, \theta_{2}\right), \varphi\left(\theta_{1}, \theta_{2}\right)\right\rangle=\frac{1}{2} \int_{-\pi}^{\pi} d \theta_{1} \int_{-\pi}^{\pi} d \theta_{2} \frac{\varphi\left(\theta_{1}, \theta_{2}\right)-\varphi(0,0)}{2-\cos \theta_{1}-\cos \theta_{2}}
$$

Again, let us emphasize that such a regularization (i) is needeed owing to the singularity at the origin and as a matter of fact, one can verify that over the disk centered at the origin and of radius $r$, the part of the integrand which contributes to the integral (5) is $O(r)$ as $r \rightarrow+0$; (ii) is proposed among other more general regularization procedure like

$$
\left\langle g\left(\theta_{1}, \theta_{2}\right), \varphi\left(\theta_{1}, \theta_{2}\right)\right\rangle=\frac{1}{2}\left(\iint_{S(\eta)} \frac{\varphi\left(\theta_{1}, \theta_{2}\right)-\varphi(0,0)}{2-\cos \theta_{1}-\cos \theta_{2}} d \theta_{1} d \theta_{2}+\iint_{[-\pi, \pi]^{2} \backslash S(\eta)} \frac{\varphi\left(\theta_{1}, \theta_{2}\right)}{2-\cos \theta_{1}-\cos \theta_{2}} d \theta_{1} d \theta_{2}\right)
$$

where $S(\eta)$ is the square $[-\eta, \eta]^{2}$ containing the singularity at the origin for any $\eta, 0<\eta \leq \pi$. The formula (6) reduces to (5) for $\eta=\pi$. Comparing (5) and (6) shows that these two finite numbers differ from the value $4 \pi^{2} g_{0} \varphi(0,0)$, with

$$
g_{0}(\eta)=\frac{1}{8 \pi^{2}} \iint_{[-\pi, \pi]^{2} \backslash S(\eta)} \frac{1}{2-\cos \theta_{1}-\cos \theta_{2}} d \theta_{1} d \theta_{2}
$$

and proves that (5) and (6) define the same generalized function on $\mathbb{R}^{2} /(2 \pi \mathbb{Z})^{2}$ up to a constant multiple of the 2-dimensional Dirac comb $\delta\left(\theta_{1}, \theta_{2}\right)$. The functional defined by (5) is called the canonical regularization of the function (4) [14] and is the one we will consider in the sequel (unless specified otherwise). We show in Appendix B that the regularized integral (6) (and the particular case (5)) is equivalent to the Hadamard 
regularization [15]. This method of regularizing a divergent integral by removing the divergent term and keeping the finite part will be relevant for the study of the continuum limit of the LGF in Section 4.

As stated above, the LGF is the Fourier coefficients

$$
G[n, m]=\left\langle g\left(\theta_{1}, \theta_{2}\right), \frac{1}{4 \pi^{2}} e^{i\left(n \theta_{1}+m \theta_{2}\right)}\right\rangle=\frac{1}{8 \pi^{2}} \int_{-\pi}^{\pi} d \theta_{1} \int_{-\pi}^{\pi} d \theta_{2} \frac{\cos n \theta_{1} \cos m \theta_{2}-1}{2-\cos \theta_{1}-\cos \theta_{2}} \quad, \quad(n, m) \in \mathbb{Z}^{2} .
$$

In particular, $G[0,0]=0$. The canonical regularization (5) defines the generalized periodic function (4) as having a zero mean value whilst the functional (6) gives the Fourier coefficients $G[n, m]+g_{0}(\eta)$. This indeed proves that the solution of (1) is in general obtained up to an arbitrary constant and confirms that any determination of parameter $\eta$ is a legitimate one for constructing the LGF. Choosing $\eta=\pi$ (i.e. the canonical regularization such that $g_{0}(\pi)=0$ ) is simply the easiest choice to be capable of computing the LGF.

\section{Lattice Green function : calculation}

The integral (8) is obviously known for a very long time and some exact and rapid evaluation can be performed using e.g. the Mathematica software for $|n|,|m| \leq 3$ (see Table 1). Of course, these numerical values agree with those already obtained e.g. in [7].

It is particularly worth noting the exact value on the lattice diagonals $n= \pm m$ may be easily computed as (see e.g. $[16,12]$ and below)

$$
G[n, n]=-\frac{1}{\pi} \sum_{l=1}^{|n|} \frac{1}{2 l-1}=-\frac{1}{2 \pi}\left(\psi\left(|n|+\frac{1}{2}\right)+\gamma+2 \log 2\right) \quad, \quad n \in \mathbb{Z} \backslash\{0\},
$$

with $\gamma$ the Euler-Mascheroni constant and $\psi(z)=\Gamma^{\prime}(z) / \Gamma(z)$ the digamma function [17, p. 258]. Hence, as emphasized in [16] progressing step by step from these values on the diagonals using the symmetry/reflection properties and the fact that $G[n, m],(n, m) \neq(0,0)$, is the mean of the values at its four nearest neighbors, all other values at any node $(n, m)$ may be thus recursively obtained. Nevertheless, we show in Appendix A that a closed-form general result for any coefficients $G[n, m]$ may be derived in terms of hypergeometric and gamma functions contrary to popular opinion that such a general result did not seem possible. It should be noted that a similar attempt was made in [18] using tools of the complex analysis to evaluate $G[n, m]$ but the obtained representation in terms of a singular generalized hypergeometric function still requiring regularization of a logarithmic singularity makes that result not very practical to use. As such, the following expression should provide a substantial reduction of Ray's representation. Indeed, it can be shown that for any $n \geq m \geq 0$,

$$
\begin{aligned}
G[n, m] & =\frac{1}{2 \pi}\left(\log 2-2 \sum_{l=1}^{n} \frac{1}{2 l-1}-\frac{m+\frac{1}{2}}{n+\frac{1}{2}}{ }_{3} F_{2}\left(1,1, m+\frac{3}{2} ; 2, n+\frac{3}{2} ;-1\right)\right) \\
& +\frac{1}{2 \pi} \sum_{k=1}^{\left\lfloor\frac{n-m}{2}\right\rfloor}(-1)^{k}\left(\begin{array}{c}
n-m \\
2 k
\end{array}\right) B\left(2 k, n-k+\frac{1}{2}\right){ }_{2} F_{1}\left(2 k, m+k+\frac{1}{2} ; n+k+\frac{1}{2} ;-1\right) .
\end{aligned}
$$

The notations and definitions are given in Appendix A. Obviously, this formula allows to retrieve in an exact form or to evaluate to arbitrary numerical precision all known results like (see [7, 19])

$$
G[ \pm 5, \pm 3]=G[ \pm 3, \pm 5]=4-\frac{499}{35 \pi} \approx-0.53819
$$

or

$$
G[ \pm 10, \pm 7]=G[ \pm 7, \pm 10]=\frac{577}{4}-\frac{20506034}{45045 \pi} \approx-0.65561,
$$

but also gives new ones like for example

$$
\begin{aligned}
G[ \pm 30, \pm 10]=G[ \pm 10, \pm 30] & =-3775513781874238568+\frac{660137345297797509134893757768588}{55655536011075 \pi} \\
& \approx-0.80704 .
\end{aligned}
$$




\begin{tabular}{|c|c|c|c|c|}
\hline \hline$|n|$ & 0 & 1 & 2 & 3 \\
\hline 0 & 0 & $-\frac{1}{4}$ & $-1+\frac{2}{\pi}$ & $-\frac{17}{4}+\frac{12}{\pi}$ \\
\hline 1 & $-\frac{1}{4}$ & $-\frac{1}{\pi}$ & $\frac{1}{4}-\frac{2}{\pi}$ & $2-\frac{23}{3 \pi}$ \\
\hline 2 & $-1+\frac{2}{\pi}$ & $\frac{1}{4}-\frac{2}{\pi}$ & $-\frac{4}{3 \pi}$ & $-\frac{1}{4}-\frac{2}{3 \pi}$ \\
\hline 3 & $-\frac{17}{4}+\frac{12}{\pi}$ & $2-\frac{23}{3 \pi}$ & $-\frac{1}{4}-\frac{2}{3 \pi}$ & $-\frac{23}{15 \pi}$ \\
\hline \hline
\end{tabular}

Table 1: Lattice Green function $G[n, m]$ in a 2-dimensional infinite square lattice.

In a general way, as already proved in [16], all these values $G[n, m]$ are of the form $a+b / \pi$ with $a$ and $b$ rational. In particular, since

$$
{ }_{3} F_{2}\left(1,1, n+\frac{3}{2} ; 2, n+\frac{3}{2} ;-1\right)=\sum_{l=0}^{+\infty} \frac{(-1)^{l}}{l+1}=\log 2,
$$

the result (9) for $G[n, n]$ is well established from the general result (10). Also, considering the following identity $[20]$

$$
{ }_{3} F_{2}(1,1, c ; 2, c+1 ;-1)=\frac{c}{2(1-c)}\left(\psi\left(\frac{c+1}{2}\right)-\psi\left(\frac{c}{2}\right)-2 \log 2\right),
$$

and the particular Lerch transcendent ([21, p. 20] and [22, p. 1391])

$$
\Phi(z)=\frac{1}{2}\left(\psi\left(\frac{z+1}{2}\right)-\psi\left(\frac{z}{2}\right)\right)=\sum_{l=0}^{+\infty} \frac{(-1)^{l}}{z+l},
$$

it is straightforward to show that the LGF at nearest sites of the diagonal is simply given by the sum

$$
G[n, n-1]=G[n-1, n]=-\frac{1}{\pi} \sum_{l=1}^{|n|} \frac{1}{2 l-1}+\frac{1}{2 \pi} \Phi\left(|n|+\frac{1}{2}\right)=-\frac{1}{\pi} \sum_{l=1}^{|n|} \frac{1}{2 l-1}+\frac{1}{2 \pi} \sum_{l=0}^{+\infty} \frac{(-1)^{l}}{|n|+\frac{1}{2}+l}
$$

for any integer $|n| \geq 1$. The interested readers might want to check that the latter formula together with (1) thus lead to the identity

$$
\psi\left(\frac{|n|}{2}+\frac{5}{4}\right)-\psi\left(\frac{|n|}{2}+\frac{1}{4}\right)=\frac{4}{2|n|+1} \quad, \quad|n| \geq 1 .
$$

in agreement with the well-known functional relation $\psi(z+1)-\psi(z)=1 / z[22$, p. 1386].

\section{Lattice Green function : asymptotics and continuum limit}

Now, let us investigate what becomes the LGF (10) far from the origin i.e. when $m$ and $n$ are large. Let

$$
r=\sqrt{n^{2}+m^{2}} \quad, \quad n=r \cos \theta \quad, \quad m=r \sin \theta .
$$


In the Euclidean plane $\mathbb{R}^{2}$ given an unit length, $r$ thus measures the distance between the lattice point of coordinates $(n, m)$ to the origin. On the diagonals, $m= \pm n$, thus $r=|n| \sqrt{2}$ and it is thereby easy to show that $[17$, p. 259]

$$
G[n, n]=-\frac{1}{2 \pi}\left(\log r+\gamma+\frac{3}{2} \log 2\right)+O\left(\frac{1}{r}\right) \quad \text { as } \quad r \rightarrow+\infty .
$$

In [12], Watson has expediently proved that such an estimate still holds true for any infinitely distant lattice point $(n, m)$ - notice that Watson has precisely given in his remarkable paper the following sharper asymptotic expansion (see also, [23]),

$$
G[n, m]=-\frac{1}{2 \pi}\left(\log r+\gamma+\frac{3}{2} \log 2-\frac{1}{12} \frac{\cos 4 \theta}{r^{2}}-\left(\frac{3}{40} \cos 4 \theta+\frac{4}{48} \cos 8 \theta\right) \frac{1}{r^{4}}+O\left(\frac{1}{r^{6}}\right)\right)
$$

- so that we set for a coming discussion,

$$
G_{\text {asympt }}[n, m]=-\frac{1}{2 \pi} \log \frac{r}{L_{0}}=-\frac{1}{2 \pi} \log \frac{\sqrt{n^{2}+m^{2}}}{L_{0}}
$$

where $L_{0}=e^{-\gamma} 2^{-3 / 2} \approx 0.198506$ is a characteristic length we have furthermore called horizon [24] related to the unit length chosen in the plane. As a result, the main consequence of all of these is that the 2 -dimensional LGF (10) is asymptotically the discretized counterpart for the fundamental solution of the planar Laplacian and exhibits the radial symmetry far enough away from the origin. Indeed, recall that the radially symmetric solution on the plane of the Poisson equation $\Delta \mathcal{G}=-\delta(\mathbf{r})$ is the logarithmic Coulomb potential

$$
\mathcal{G}(\mathbf{r})=-\frac{1}{2 \pi} \log \frac{|\mathbf{r}|}{L}
$$

where the horizon $L$ is an arbitrarily chosen positive length relevant to the physical scale of the problem allowing us to fix a zero potential at finite distance of the origin (precisely, on the circle $|\mathbf{r}|=L>0$ ) but also more deeply, is needeed to report on the scale invariance of the fundamental solution $\mathcal{G}$ and to adimensionalize constructively the argument of the logarithm [25]. Usually, one sets $R=1$ without any substantive reason except for convenience, but what precedes has shown that choosing here the particular value $L=L_{0}<1$ in the first instance allows matching accurately the asymptotic result (13) to the LGF $(10)$.

In addition, let us notice considering (12) that the relative error

$$
\varepsilon(r)=\left|\frac{G[n, m]-G_{\text {asympt }}[n, m]}{G[n, m]}\right|
$$

is $O\left(1 /\left(r^{2} \log r\right)\right)$, and numerical evaluations indicate that this error is already less than $1 \%$ when $r>2$ (see Figure 1 and Table 2).

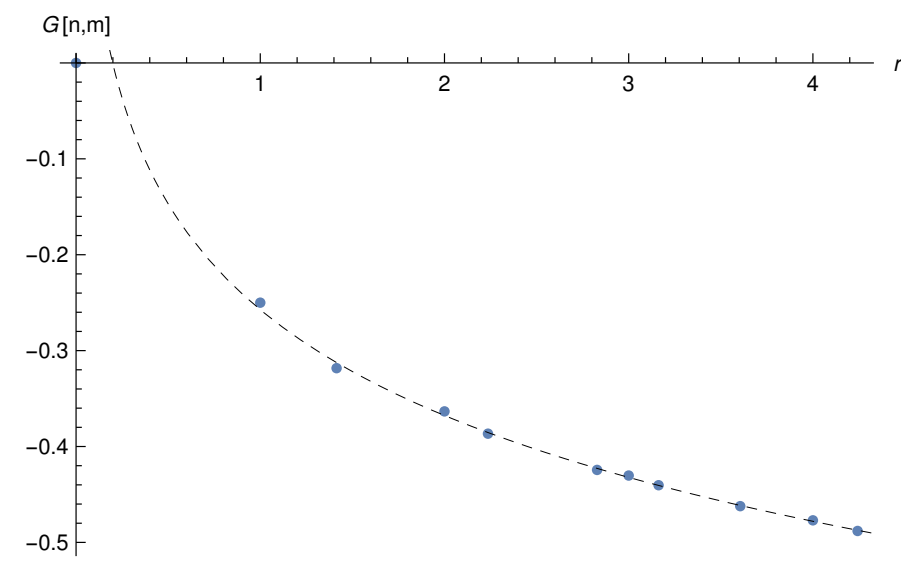

Figure 1: Plots of the LGF $G[n, m]$ (dotted) and the logarithmic estimate $G_{\text {asympt }}[n, m]=(-1 / 2 \pi) \log \left(r / L_{0}\right)$ (dashed) as function of the distance $r=\sqrt{n^{2}+m^{2}}$ ranging from 0 to $3 \sqrt{2}$. 


\begin{tabular}{|c|c|c|c|c|c|c|c|c|c|c|}
\hline \hline$r_{(n, m)}$ & $1_{(1,0)}$ & $\sqrt{2}_{(1,1)}$ & $2_{(2,0)}$ & $\sqrt{5}_{(2,1)}$ & $\sqrt{8}_{(2,2)}$ & $3_{(3,0)}$ & $\sqrt{10}_{(3,1)}$ & $\sqrt{13}_{(3,2)}$ & $4_{(4,0)}$ & $\sqrt{18}_{(3,3)}$ \\
\hline$\varepsilon(r)_{\%}$ & 2.94 & 1.82 & 1.18 & 0.31 & 0.37 & 0.44 & 0.04 & 0.16 & 0.21 & 0.15 \\
\hline \hline
\end{tabular}

Table 2: Percent relative error between the LGF $G[n, m]$ and the logarithmic estimate $G_{\text {asympt }}[n, m]$ for $r=$ $\sqrt{n^{2}+m^{2}}$ ranging from 1 to $3 \sqrt{2}$ unit lengths. This error is asymptotically zero as $r$ tends to infinity.

Hence, one may reasonably claim that the horizon $L_{0}$ characterizes in the plane $\mathbb{R}^{2}$ the radius of a disk centered at the origin/singularity of the logarithmic potential (14): $(i)$ in which the lattice discretization assigns the finite regularized value $G[0,0]=0 ;(i i)$ and outside of which the approximation $G[n, m] \approx \mathcal{G}(\mathbf{r}=$ $(n, m))$ i.e.

$$
G[n, m] \approx-\frac{1}{2 \pi} \log \frac{\sqrt{n^{2}+m^{2}}}{L_{0}} \quad, \quad(n, m) \neq(0,0)
$$

holds with a sufficient accuracy for most of physical applications. Additionally, it is of great importance to note that owing the scale invariance of the continuous fundamental solution $\mathcal{G}$, all the foregoing remain true if we set

$$
\mathbf{r}=(n a, m a) \quad, \quad r=a \sqrt{n^{2}+m^{2}} \quad, \quad \forall a>0
$$

and take a circular cut-off of radius (or horizon) $a L_{0}$. This amounts to modify the physical scale of the problem and consider the discretization on the 2-lattice $(a \mathbb{Z})^{2}$ of spacing $a$. Consequently, for a given $\mathbf{r} \in \mathbb{R}^{2} \backslash\{\mathbf{0}\}$, taking the limit $a \rightarrow+0$ implies $\sqrt{n^{2}+m^{2}}$ infinitely large and de facto positions ourselves in the asymptotic conditions. So that we are surprisingly led to conclude that the continuum (or scaling) limit at point $\mathbf{r} \neq \mathbf{0}$ of the LGF (10) when defined for the 2 -lattice $(a \mathbb{Z})^{2}$ should be

$$
\lim _{\eta \rightarrow+0}-\frac{1}{2 \pi} \log \frac{|\mathbf{r}|}{\eta}, \quad \eta=a L_{0}
$$

i.e. a logarithmic potential with a null horizon, which is not obviously an acceptable result, and simply means that the continuum limit for the 2-lattice $(a \mathbb{Z})^{2}$ as previously addressed cannot be summed up by a simple rescaling of $a$ to zero. Indeed, the crux of the problem here lies in the prior condition $G[0,0]=0$ that is conserved by rescaling of $a$, thus precluding the continuum limit to mimic the logarithmic divergence at the origin. Such a difficulty is well-known in many areas of theoretical physics (classical and quantum field theories for instance) where the discretization on lattice are implemented for regularization of all the divergences due to singularities in the continuum as for instance when dealing with ultraviolet divergences in perturbative quantum field theory (see e.g. $[1,8,9]$ ). Hence, in order to draw conclusions about the solutions for the continuous case, a renormalization procedure is in fine needed to restore as much as possible the correct behaviour [26].

In our case, writing (16) as

$$
-\frac{1}{2 \pi} \log \frac{|\mathbf{r}|}{L}-g_{0}(+0)
$$

( $L$ being an arbitrary positive constant) evidences the divergent term (see (28), Appendix B)

$$
g_{0}(+0)=\lim _{\eta \rightarrow+0}-\frac{1}{2 \pi} \log \frac{\eta}{L}
$$

which may be interpreted as the self-potential of the origin point source. The lattice regularization has simply removed this divergent term by assigning the null value at the origin, the renormalization procedure reinstating this self-potential to restore the correct behaviour. As a result, the renormalized scaling limit of the LGF $G[n, m]$ may be derived by substituting $g_{0}(+0)$ for $G[0,0]=0$ so that the renormalized LGF formally must read

$$
G_{\text {renorm }}[n, m]=G[n, m]+g_{0}(+0)
$$

whose continuum limit is as expected the logarithmic potential (14) owning the scale and rotational invariance. 


\subsection{An application}

a. Consider an infinite square lattice formed from equal resistors $R$ with nodes $(n, m) \in \mathbb{Z}^{2}$ and assume a current $I$ flowing from the origin node $(0,0)$ to a distant node $\left(n_{0}, m_{0}\right)$.

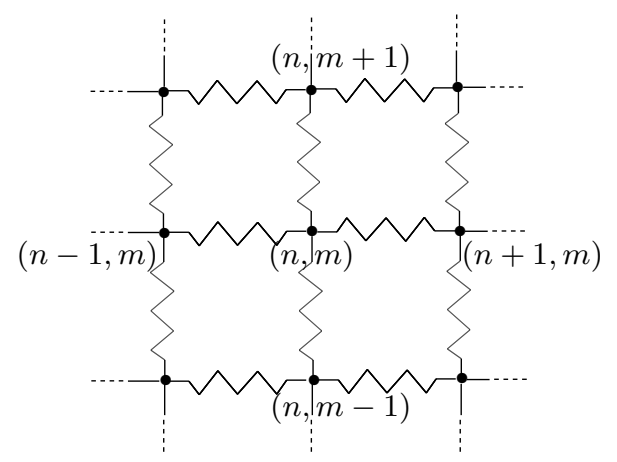

The electric potential $V[n, m]$ at node $(n, m)$ is thus solution of the partial difference equation

$$
\begin{aligned}
\frac{V[n-1, m]-V[n, m]}{R}+ & \frac{V[n+1, m]-V[n, m]}{R}+\frac{V[n, m-1]-V[n, m]}{R} \\
& +\frac{V[n, m+1]-V[n, m]}{R}=-I\left(\delta[n, m]-\delta\left[n-n_{0}, m-m_{0}\right]\right)
\end{aligned}
$$

i.e. $\Delta V[n, m]=-R I\left(\delta[n, m]-\delta\left[n-n_{0}, m-m_{0}\right]\right)$. Hence, from $(1)$, the exact value of the potential is

$$
V[n, m]=R I\left(G[n, m]-G\left[n-n_{0}, m-m_{0}\right]\right)
$$

where the LGF $G$ is given by (10). Since $G[0,0]=0$ and given the symmetry properties, it is worth to notice that

$$
V\left[n_{0}, m_{0}\right]=-V[0,0]=R I G\left[n_{0}, m_{0}\right] .
$$

Therefore, the two-point resistance between these two nodes is defined as

$$
R_{\mathbf{0}, \mathbf{r}_{0}}=\frac{V[0,0]-V\left[n_{0}, m_{0}\right]}{I}=-2 R G\left[n_{0}, m_{0}\right]
$$

By way of example, the exact value of the resistance between the origin and one of the nodes $( \pm 30, \pm 10)$ is

$$
2 R\left(3775513781874238568-\frac{660137345297797509134893757768588}{55655536011075 \pi}\right) \approx 1.61408 R .
$$

Obviously, application of (19) and (10) allows to find all known results both theoretical and experimental (see e.g. $[7,6,11])$.

b. Let us investigate now the continuum limit of the previous resistor network considering the homogeneous conducting plane $\mathbb{R}^{2}$ of (free length scale) conductivity $\sigma_{2}=1 / R$ (in $\Omega^{-1}$ ), a current $I$ flowing from the origin to point $\mathbf{r}_{0}$. As it is known [25, 27], some key results are well established : the electric potential at point $\mathbf{r}$ due to the steady flow of charges is solution of the Poisson equation $\Delta V=\left(-I / \sigma_{2}\right)\left(\delta(\mathbf{r})-\delta\left(\mathbf{r}-\mathbf{r}_{0}\right)\right)$ and may be expressed as (see (14)),

$$
V(\mathbf{r})=\frac{I}{\sigma_{2}}\left(\mathcal{G}(\mathbf{r})-\mathcal{G}\left(\mathbf{r}-\mathbf{r}_{0}\right)\right)=\frac{I}{2 \pi \sigma_{2}}\left(\log \frac{\left|\mathbf{r}-\mathbf{r}_{0}\right|}{L}-\log \frac{|\mathbf{r}|}{L}\right)=\frac{I}{2 \pi \sigma_{2}} \log \frac{\left|\mathbf{r}-\mathbf{r}_{0}\right|}{|\mathbf{r}|}
$$

exhibiting two singularities at current input and output points, thus making impossible to define the twopoint resistance $R_{\mathbf{0}, \mathbf{r}_{0}}$ by the common formula

$$
R_{\mathbf{0}, \mathbf{r}_{0}}=\frac{V(\mathbf{0})-V\left(\mathbf{r}_{0}\right)}{I}=\frac{2}{\sigma_{2}}\left(\mathcal{G}(\mathbf{0})-\mathcal{G}\left(\mathbf{r}_{0}\right)\right) .
$$


to be compared with (19). The utility of lattice discretization technique is here evident since the renormalization procedure discussed in the previous section can give instead a mathematical sense to (20): indeed, the renormalized scaling limit of (19) is simply

$$
R_{\mathbf{0}, \mathbf{r}_{0}}=\frac{1}{\pi \sigma_{2}} \log \frac{\left|\mathbf{r}_{0}\right|}{L} \quad, \quad L \text { a constant }
$$

a result identical to the one obtained in [25] where the regularization procedure consisted in removing the Coulomb singularity from (20) using an electrostatic energy-type argument. Nevertheless, this is a result which leaves us unsatisfied because the horizon $L$ can be arbitrarily fixed and thus, leads us to the surprising conclusion that the two-point resistance between any pair of current input and output points in the infinite conducting plane is indeterminable in contrast to the discretized case of the 2-dimensional resistor network. It is rather curious for instance that choosing $L=\left|\mathbf{r}_{0}\right|$, one may obtain a zero resistance! All of this appear to reflect an universal specific characteristic of the 2-dimensional space in which the electrical conduction could not be limited, a property that cannot be found in any other dimensionality. This also means possibly, indeed roughly from the point of view of classical fields theory, that the intriguing features observed in electric properties of 2D materials like graphene or any 2D crystalline matter [28, 29] may be nothing but the very evidence of the only algebraic dimension of the conducting flatland regardless of the accuracy and the refinement of physical models describing the conduction mechanism.

\section{Acknowledgments}

The author is grateful to the reviewers for their constructive comments and suggestions.

\section{Appendix A : Exact value of $G[n, m]$}

The exact value of the LGF $G[n, m]$ given by the double integral (8) is derived in this Appendix. First, using the change of variables $\theta_{1}=u+v$ and $\theta_{2}=u-v$, this integral reads,

$$
G[n, m]=\frac{1}{2 \pi^{2}} \int_{0}^{\pi / 2} d v\left(\cos (n-m) v \int_{0}^{\pi} \frac{\cos (n+m) u}{1-\cos u \cos v} d u-\int_{0}^{\pi} \frac{1}{1-\cos u \cos v} d u\right)
$$

The two integrals in brackets are evaluated without difficulties using the following result :

$$
\int_{0}^{\pi} \frac{\cos k u}{1-\alpha \cos u} d u=\frac{\pi}{\alpha^{k}} \frac{\left(1-\sqrt{1-\alpha^{2}}\right)^{k}}{\sqrt{1-\alpha^{2}}} \quad, \quad|\alpha|<1 \quad, \quad k \geq 0
$$

so that

$$
G[n, m]=\frac{1}{2 \pi} \int_{0}^{\pi / 2} \frac{d v}{\sin v}\left(\cos (n-m) v\left(\frac{1-\sin v}{\cos v}\right)^{n+m}-1\right) .
$$

Then, setting $x=\cos v$ and introducing the Chebyshev polynomial of the first $\operatorname{kind} T_{k}(\cos v)=\cos k v$ yield to the expression

$$
G[n, m]=\frac{1}{2 \pi} \int_{0}^{1} \frac{d x}{1-x^{2}}\left(\frac{x^{n+m} T_{|n-m|}(x)}{\left(1+\sqrt{1-x^{2}}\right)^{n+m}}-1\right) .
$$

Now, assuming $0 \leq m \leq n$ throughout the Appendix (otherwise, simply switch the roles of $n$ and $m$ or/and use the symmetry properies), let us remark that the product $x^{n+m} T_{n-m}(x)$ is explicitly the polynomial of degree $2 n$

$$
x^{n+m} T_{n-m}(x)=x^{2 n}+\sum_{k=1}^{\left\lfloor\frac{n-m}{2}\right\rfloor}(-1)^{k}\left(\begin{array}{c}
n-m \\
2 k
\end{array}\right)\left(x^{2}\right)^{n-k}\left(1-x^{2}\right)^{k}
$$

allowing to write $G[n, m]$ as the finite sum of convergent integrals

$$
G[n, m]=\frac{1}{2 \pi} \int_{0}^{1} \frac{d x}{1-x^{2}}\left(\frac{x^{2 n}}{\left(1+\sqrt{1-x^{2}}\right)^{n+m}}-1\right)+\frac{1}{2 \pi} \sum_{k=1}^{\left\lfloor\frac{n-m}{2}\right\rfloor}(-1)^{k}\left(\begin{array}{c}
n-m \\
2 k
\end{array}\right) \int_{0}^{1} \frac{\left(x^{2}\right)^{n-k}\left(1-x^{2}\right)^{k-1}}{\left(1+\sqrt{1-x^{2}}\right)^{n+m}} d x
$$


the first integral being the single term where the regularization is needed. Making finally the change of variable $t=\sqrt{1-x^{2}}$, one obtains in an easier tractable form,

$$
G[n, m]=\frac{1}{2 \pi} \int_{0}^{1} \frac{d t}{t \sqrt{1-t^{2}}}\left(\frac{(1-t)^{n}}{(1+t)^{m}}-1\right)+\frac{1}{2 \pi} \sum_{k=1}^{\left\lfloor\frac{n-m}{2}\right\rfloor}(-1)^{k}\left(\begin{array}{c}
n-m \\
2 k
\end{array}\right) \int_{0}^{1} t^{2 k-1} \frac{(1-t)^{n-k-1 / 2}}{(1+t)^{m+k+1 / 2}} d t .
$$

The second integral is related to the Euler's fundamental representation of Gaussian hypergeometric functions [21] and is straightforwardly found having the exact value (see e.g. [22, formula 8, p. 315])

$$
\int_{0}^{1} t^{2 k-1} \frac{(1-t)^{n-k-1 / 2}}{(1+t)^{m+k+1 / 2}} d t=B\left(2 k, n-k+\frac{1}{2}\right){ }_{2} F_{1}\left(2 k, m+k+\frac{1}{2} ; n+k+\frac{1}{2} ;-1\right)
$$

(where $B$ is the beta function and ${ }_{2} F_{1}$ the Gauss hypergeometric function) while the first one (which represents the regularization of a divergent integral owing to the singularity at $t=0$ ) may be rewritten as

$$
\begin{aligned}
\int_{0}^{1} \frac{d t}{t \sqrt{1-t^{2}}}\left(\frac{(1-t)^{n}}{(1+t)^{m}}-1\right) & =\lim _{\epsilon \rightarrow 0+}\left(\int_{\epsilon}^{1} \frac{d t}{t} \frac{(1-t)^{n-1 / 2}}{(1+t)^{m+1 / 2}}+\log \epsilon\right)+\int_{0}^{1} \frac{d t}{t}\left(1-\frac{1}{\sqrt{1-t^{2}}}\right) \\
& =f_{0}^{1} \frac{d t}{t} \frac{(1-t)^{n-1 / 2}}{(1+t)^{m+1 / 2}}-\log 2 .
\end{aligned}
$$

The slashed integral notation here indicates the Hadamard finite part of integral (26) as $k$ tends to 0 [15, 30], which can be simply derived considering the power series expansion of the RHS around $k=0$. Indeed, we have on the one hand [17, p. 258]

$$
B\left(2 k, n-k+\frac{1}{2}\right)=\frac{1}{2 k}-\gamma-\psi\left(n+\frac{1}{2}\right)+O(k)=\frac{1}{2 k}+2 \log 2-2 \sum_{l=1}^{n} \frac{1}{2 l-1}+O(k)
$$

with $\gamma$ the Euler-Mascheroni constant and $\psi(z)$ the digamma function, whilst on the other hand via a Taylor series,

$$
{ }_{2} F_{1}\left(2 k, m+k+\frac{1}{2} ; n+k+\frac{1}{2} ;-1\right)=1+2 k \partial_{a} F\left(0, m+\frac{1}{2} ; n+\frac{1}{2} ;-1\right)+O(k)^{2}
$$

where $\partial_{a} F$ means a differentiation with respect to the first parameter of the hypergeometric function $F=$ ${ }_{2} F_{1}(a, b ; c ; z)$ ( $F$ is here an entire analytic function of $k[21]$ and we recall that ${ }_{2} F_{1}(0, b ; c ; z)=1$ and $\partial_{b} F(0, b ; c ; z)=\partial_{c} F(0, b ; c ; z)=0$ (see e.g. [31,32] for a modern and comprehensive investigation of these derivatives and others)). Instead, the derivative $\partial_{a} F$ deserves particular attention: considering the Gauss hypergeometric function written as

$$
F={ }_{2} F_{1}(a, b ; c ; z)=\sum_{l=0}^{+\infty} \frac{(a)_{l}(b)_{l}}{(c)_{l}} \frac{z^{l}}{l !}
$$

where $(\alpha)_{l}$ denotes the so-called Pochhammer symbol $(\alpha)_{l}=\Gamma(\alpha+l) / \Gamma(\alpha)$ of derivative

$$
\partial_{\alpha}(\alpha)_{l}=(\alpha)_{l}(\psi(\alpha+l)-\psi(\alpha)),
$$

it follows that

$$
\partial_{a} F(a, b ; c ; z)=\sum_{l=1}^{+\infty} \frac{(a)_{l}(b)_{l}}{(c)_{l}}(\psi(a+l)-\psi(a)) \frac{z^{l}}{l !} .
$$

When the parameter $a$ tends to zero, $(a)_{l}$ tends to zero for $l \geq 1$ and so the product $(a)_{l} \psi(a+l)$, but

$$
(a)_{l} \psi(a)=\Gamma(a+l) \frac{\Gamma^{\prime}(a)}{\Gamma^{2}(a)}=-\Gamma(a+l) \frac{d}{d a}\left(\frac{1}{\Gamma(a)}\right)
$$

tends to $-\Gamma(l)$ since $1 / \Gamma(a)=a+O(a)^{2}[17$, p. 256]. Therefore,

$$
\begin{aligned}
\partial_{a} F\left(0, m+\frac{1}{2} ; n+\frac{1}{2} ;-1\right) & =\sum_{l=1}^{+\infty} \frac{(-1)^{l}}{l} \frac{\left(m+\frac{1}{2}\right)_{l}}{\left(n+\frac{1}{2}\right)_{l}}=-\sum_{l=0}^{+\infty} \frac{\left(m+\frac{1}{2}\right)_{l+1}}{\left(n+\frac{1}{2}\right)_{l+1}} \frac{(-1)^{l}}{l+1} \\
& =-\frac{m+\frac{1}{2}}{n+\frac{1}{2}} \sum_{l=0}^{+\infty} \frac{\left(m+\frac{3}{2}\right)_{l}(1)_{l}(1)_{l}}{\left(n+\frac{3}{2}\right)_{l}(2)_{l}} \frac{(-1)^{l}}{l !}
\end{aligned}
$$


using the identities $(1)_{l}=l$ ! and $(\alpha)_{l+1}=\alpha(\alpha+1)_{l}$. Finally, we notice that the latter sum may be interpreted as the series representation for argument $z=-1$ of the hypergeometric function ${ }_{3} F_{2}(1,1, m+3 / 2 ; 2, n+3 / 2 ; z)$.

Accordingly, retaining for the product (26) the finite term when $k=0$, it results the Hadamard finite part

$$
f_{0}^{1} \frac{d t}{t} \frac{(1-t)^{n-1 / 2}}{(1+t)^{m+1 / 2}}=2 \log 2-2 \sum_{l=1}^{n} \frac{1}{2 l-1}-\frac{m+\frac{1}{2}}{n+\frac{1}{2}}{ }_{3} F_{2}\left(1,1, m+\frac{3}{2} ; 2, n+\frac{3}{2} ;-1\right)
$$

and whence the result (10).

\section{Appendix B : Hadamard regularization of $g\left(\theta_{1}, \theta_{2}\right)$}

Consider the canonical regularization of $g\left(\theta_{1}, \theta_{2}\right)$ defined by the functional (5) we recast as follows,

$$
\begin{aligned}
\left\langle g\left(\theta_{1}, \theta_{2}\right), \varphi\left(\theta_{1}, \theta_{2}\right)\right\rangle= & \lim _{\eta \rightarrow 0+}\left(\frac{1}{2} \iint_{[-\pi, \pi]^{2} \backslash S(\eta)} \frac{\varphi\left(\theta_{1}, \theta_{2}\right)-\varphi(0,0)}{2-\cos \theta_{1}-\cos \theta_{2}} d \theta_{1} d \theta_{2}\right) \\
& =\lim _{\eta \rightarrow 0+}\left(\frac{1}{2} \iint_{[-\pi, \pi]^{2} \backslash S(\eta)} \frac{\varphi\left(\theta_{1}, \theta_{2}\right)}{2-\cos \theta_{1}-\cos \theta_{2}} d \theta_{1} d \theta_{2}-8 \pi^{2} \varphi(0,0) g_{0}(\eta)\right)
\end{aligned}
$$

where $g_{0}$ is given by $(7)$ and $S(\eta)$ is the square $[-\eta, \eta]^{2}$ containing the singularity at the origin for any $\eta, 0<\eta \leq \pi$.

The term $g_{0}(\eta)$ is logarithmically divergent when $\eta \rightarrow+0$. Precisely, one could show that

$$
g_{0}(\eta)=-\frac{1}{2 \pi} \log \eta+O(1) \quad \text { as } \eta \rightarrow+0
$$

i.e.

$$
g_{0}(\eta) \underset{\eta \rightarrow+0}{\sim}-\frac{1}{2 \pi} \log \frac{\eta}{L} \quad, \quad L \text { a constant. }
$$

On the one hand, it should be noted that the constant $L$ is not arbitrary but must have an appropriate value (say, $L=L_{1}$ ) in order that the doubly periodic function $g$ has a zero mean value. On the other hand, the logarithmic term (28) is precisely the divergent part of the integral (27) which is dropped. As a result, this shows that the canonical regularization is equivalent to the following Hadamard finite part integral

$$
\begin{aligned}
\left\langle g\left(\theta_{1}, \theta_{2}\right), \varphi\left(\theta_{1}, \theta_{2}\right)\right\rangle & =\frac{1}{2} \underset{[-\pi, \pi]^{2}}{\int-\cos \theta_{1}-\cos \theta_{2}} d \theta_{1} d \theta_{2} \\
& =\lim _{\eta \rightarrow+0} \frac{1}{2}\left(\iint_{[-\pi, \pi]^{2} \backslash S(\eta)} \frac{\varphi\left(\theta_{1}, \theta_{2}\right)}{2-\cos \theta_{1}-\cos \theta_{2}} d \theta_{1} d \theta_{2}+4 \pi \varphi(0,0) \log \frac{\eta}{L_{1}}\right) .
\end{aligned}
$$

Moreover, replacing $L_{1}$ with $L \neq L_{1}$ results in the Hadamard regularization related to the functional (6) which defines the same generalized function $g$ up to the Dirac comb $2 \pi \log \left(L_{1} / L\right) \delta\left(\theta_{1}, \theta_{2}\right)$.

Regarding the LGF, these Fourier coefficients here read

$$
\begin{aligned}
G[n, m] & =\left\langle g\left(\theta_{1}, \theta_{2}\right), \frac{1}{4 \pi^{2}} e^{i\left(n \theta_{1}+m \theta_{2}\right)}\right\rangle \\
& =\lim _{\eta \rightarrow+0}\left(\frac{1}{8 \pi^{2}} \iint_{[-\pi, \pi]^{2} \backslash S(\eta)} \frac{\cos n \theta_{1} \cos m \theta_{2}}{2-\cos \theta_{1}-\cos \theta_{2}} d \theta_{1} d \theta_{2}+\frac{1}{2 \pi} \log \frac{\eta}{L_{1}}\right)
\end{aligned}
$$

with $G[0,0]=0$. The term (28) in the limit $\eta \rightarrow+0$ is heuristically the key element in the renormalization procedure needed to obtain the continuum limit of the LGF $G[n, m]$ when defined on the 2-lattice $(a \mathbb{Z})^{2}$ of infinitely small spacing $a$ (see (17)). In addition, let us emphasize that such a divergent term corresponds in 2-D potential theory to the self-potential of an origin point source whose removal from certain divergent integrals involved in the evaluation of lattice sums is ubiquitous in many physical problems like e.g. the Madelung constant in physics of crystal (see e.g. [33] for more details). 


\section{References}

[1] M. Creutz. Quark, Gluons, and Lattices. Cambridge Monographs on Mathematical Physics. Cambridge University Press, 1983.

[2] H. J. Rothe. Lattice Gauge Theories. World Scientific, 4th edition, 2012.

[3] C. Itzykson and J.-M. Drouffe. Statistical field theory, volume 1 of Cambridge monographs on mathematical physics. Cambridge University Press, 1989.

[4] B. D. Hughes. Random Walks and Random Environments: Random Walks, volume 1. Oxford University Press, USA, 1995.

[5] P. G. Doyle and L. Snell. Random Walks and Electric Networks (Carus Mathematical Monographs). Mathematical Assn of America, First Printing edition, December 1984.

[6] J. Cserti. Application of the lattice Green's function for calculating the resistance of an infinite network of resistors. American Journal of Physics, 68(10):896-906, 2000.

[7] D. Atkinson and F. J. van Steenwijk. Infinite resistive lattices. American Journal of Physics, 67(6):486492, 1999.

[8] S. K. Adhikari, T. Frederico, and R. M. Marinho. Lattice discretization in quantum scattering. Journal of Physics A: Mathematical and General, 29(22):7157-7163, 1996.

[9] Z. Maassarani. Series expansions for lattice Green functions. Journal of Physics A: Mathematical and General, 33(32):5675-5691, 2000.

[10] P.A. Martin. Discrete scattering theory: Green's function for a square lattice. Wave Motion, 43(7):619 $-629,2006$.

[11] J. H. Asad, A. Sakaji, R. S. Hijjawi, and J. M. Khalifeh. On the resistance of an infinite square network of identical resistors - theoretical and experimental comparison. The European Physical Journal B Condensed Matter and Complex Systems, 52:365-370, 2006.

[12] E. J. Watson. Infinite regular electrical networks. European Journal of Applied Mathematics, 16(5):555$567,2005$.

[13] M.L. Glasser and J. Boersma. Exact values for the cubic lattice Green functions. Journal of Physics A: Mathematical and General, 33(28):5017, 2000.

[14] I. M. Gel'fand and G. E. Shilov. Generalized functions. Properties and operations, volume 1. Academic Press, 1st edition, 1964.

[15] L. Blanchet and G. Faye. Hadamard regularization. Journal of Mathematical Physics, 41(11):7675-7714, 2000.

[16] D. Cameron. The square grid of unit resistors. The Mathematical Scientist, 11:75 - 82, 1986.

[17] M. Abramowitz and I. Stegun. Handbook of Mathematical Functions. Dover Publications, 1965.

[18] K. Ray. Green's function on lattices, arXiv:1409.7806v1 [math-ph]. 2014.

[19] G. Venezian. On the resistance between two points on a grid. American Journal of Physics, 62(11):10001004, 1994.

[20] WolframResearch. http://functions.wolfram.com/07.27.03.0108.01.

[21] A. Erdélyi et al. H. Bateman. Higher transcendental functions, volume 1. McGraw-Hill, 1953.

[22] I. S. Gradshteyn and I. M. Ryzhik. Table of Integrals, Series, and Products. Academic Press, 2000.

[23] P.-G. Martinsson and G. J. Rodin. Asymptotic expansions of lattice Green's functions. Proceedings of the Royal Society A: Mathematical, Physical and Engineering Sciences, 458:2609-2622, 2002. 
[24] M. Mamode. Fundamental solution of the Laplacian on flat tori and boundary value problems for the planar Poisson equation in rectangles. Boundary Value Problems, 1:221, 2014.

[25] M. Mamode. Calculation of two-point resistances for conducting media needs regularization of Coulomb singularities. The European Physical Journal Plus, 134:559, 2019.

[26] M. Creutz, L. Jacobs, and C. Rebbi. Monte Carlo computations in lattice gauge theories. Physics Reports, 95(4):201 - 282, 1983.

[27] P. M. Chaikin and T. C. Lubenskii. Principles of condensed matter physics. Cambridge Univ. Press, Cambridge, 1995.

[28] A. Geim and A. MacDonald. Graphene: Exploring carbon flatland. Physics Today, 60(8):35, 2007.

[29] J. L. Miller. Unconventional superconductivity discovered in graphene bilayers. Physics Today, 71(5):15, 2018.

[30] J. Hadamard. Le problème de Cauchy et les équations aux dérivées partielles linéaires hyperboliques. Hermann, Paris, 1932.

[31] J. Fröhlich. Parameter derivatives of the Jacobi polynomials and the Gaussian hypergeometric function. Integral Transforms and Special Functions, 2(4):253-266, 1994.

[32] L. U. Ancarani and G. Gasaneo. Derivatives of any order of the Gaussian hypergeometric function 2F1(a,b;c;z) with respect to the parameters a,b and c. Journal of Physics A: Mathematical and Theoretical, 42(39):395208, 2009.

[33] J.M. Borwein, M.L. Glasser, R.C. McPhedran, J.G. Wan, and I.J. Zucker. Lattice Sums Then and Now. Encyclopedia of Mathematics and its Applications. Cambridge University Press, 2013. 